\title{
A Note on CCMV Portfolio Optimization Model with Short Selling and Risk-neutral Interest Rate
}

\author{
Tahereh Khodamoradi, Maziar Salahi*, Ali Reza Najafi \\ Department of Applied Mathematics, Faculty of Mathematical Sciences, University of Guilan, Iran
}

\begin{abstract}
In this paper, first, we discuss some drawbacks of the cardinality constrained mean-variance (CCMV) portfolio optimization with short selling and risk-neutral interest rate when the lower and upper bounds of the assets contributions are $-\frac{1}{K}$ and $\frac{1}{K}$ ( $\mathrm{K}$ denotes the number of assets in portfolio). Second, we present an improved variant using absolute returns instead of the returns to include short selling in the model. Finally, some numerical results are provided using the data set of the S\&P 500 index, Information Technology, and the MIBTEL index in terms of returns and Sharpe ratios to compare the proposed models with those in the literature.
\end{abstract}

Keywords CCMV model; Short selling; Risk-neutral interest rate; Quadratic optimization.

AMS 2010 subject classifications 90C11, 91G10

DOI: $10.19139 /$ soic-2310-5070-890

\section{Introduction}

Portfolio selection is one of the most relevant and studied topics in finance. Harry Markowitz [24] presented the main model of portfolio optimization in 1952. In his model, the main idea is to minimize the risk for a given level of expected return or maximize the expected return for a given level of risk. Some extensions of his model are including transaction costs [26], multi-period optimization [19], and the cardinality constraint which considers a limit on the number of assets in the portfolio and the quantity constraints which restrict the proportion of each asset in the portfolio to lie between given lower and upper bounds. Cardinality Constrained Mean-Variance (CCMV) portfolio optimization model is a mixed integer quadratic optimization problem, which is NP-hard compared to MV model which is polynomially solvable. Chang et al. [5] have implemented three local search heuristics, namely Simulated Annealing (SA), Tabu search (TS) and a basic Genetic Algorithm (GA) to find the cardinality constrained efficient frontier. They showed that, the GA heuristic is better than the SA heuristic, which in turn is better than the TS heuristic. In 2008, Shaw et al. [28] used the Lagrangian relaxation to make lower bounds for such problems. Xie et al. [29] adopted a randomized algorithm to find good feasible solutions to CCMV problem with some quality guarantee. Further efforts to develop efficient algorithms for solving CCMV model can be found in Dueck and Winker [9], Bienstock [3], Maringer and Kellerer [23], Derigs and Nickel [8], Li et al. [20], Bertsimas and Shioda [1] and Gao and Li [11].

In Markowitz's model, short selling strategy has not been taken into account, which is usually done by borrowing the assets and selling them. Short selling can benefit an investor by yielding extra returns. Investors apply short selling when they believe that the price of the assets will decline. It is well known that imposing short selling improves the performance of the mean-variance portfolio, see for example [10,16]. Lintner [21] was the first to

\footnotetext{
*Correspondence to: Maziar Salahi (Email: salahim@ guilan.ac.ir). Department of Applied Mathematics, Faculty of Mathematical Sciences, University of Guilan, Rasht, Iran
}

ISSN 2310-5070 (online) ISSN 2311-004X (print)

Copyright (C) 2020 International Academic Press 
present a more realistic model of short selling in portfolio optimization theory. Later, Kwan [18] discussed the normative and market equilibrium perspective of portfolio selection with short selling. Also, Zhang et al. [30] extended the MV model for different interest rates for borrowing and lending. Another factor that can be used with short selling strategy is the risk-neutral interest rate. It represents the interest an investor would expect from an absolutely risk-neutral investment over a specified period of time, see for example Birge et al. [4], Bielecki et al. [2], and Maringer [22]. Jacobs et al. [14,15] used short selling and risk-neutral interest rate together. Also, in 2013 Gao and Li also [12] studied CCMV with and without short selling and risk-neutral interest rate.

A strategy to split wealth uniformly between the available investment is the so called naive strategy. In this strategy, the weight $\frac{1}{N}$ of wealth is allocated to each of the $N$ assets. It does not involve any optimization or estimation and completely ignores the data. There are two reasons for using this strategy as a benchmark: First, it is easy to implement. Second, despite the sophisticated models developed and the advances in approaches for estimating the parameters of models, investors use such simple allocation rules for allocating their wealth across assets. Due to estimation errors, Jobson and Korkie [17] stated that the naive approach can outperform the Markowitz model. In 2007, DeMiguel et al. [7] used the naive strategy as a benchmark in a rolling horizon setting and compared it with several portfolio optimization models. They concluded that optimizing models are expected to outperform the naive strategy if they have three cases: (i) the number of assets is small; (ii) the estimation window is long; and (iii) the true Sharpe ratio of the mean-variance efficient portfolio is remarkably higher than that of the naive strategy. Also, Pflug et al. [27] demonstrated that the naive strategy is sensible when the agent has a sufficiently high degree of model uncertainty in the form of ambiguous loss distributions.

In this paper, first we study the CCMV model with short selling and risk-neutral interest rate when the lower bounds on the assets contribution are $-\frac{1}{K}$ and upper bounds are $\frac{1}{K}$, where $K$ is the number of assets in the portfolio. A drawback of this model is discussed. Then, we propose an improved variant of it using absolute returns instead of the returns to include short selling. Some numerical results are provided to compare the improved model with the existing ones.

\section{CCMV model with short selling and risk-neutral interest rate}

The CCMV model for portfolio optimization is as follows:

$$
\begin{array}{ll}
\min _{x, z} & \lambda\left(\sum_{i=1}^{N} \sum_{j=1}^{N} x_{i} x_{j} \sigma_{i, j}\right)-(1-\lambda)\left(\sum_{i=1}^{N} x_{i} \mu_{i}\right) \\
\text { s.t. } & \sum_{i=1}^{N} x_{i}=1, \\
& \sum_{i=1}^{N} z_{i}=K, \\
& \varepsilon_{i} z_{i} \leq x_{i} \leq \delta_{i} z_{i}, \quad i=1, \ldots, N, \\
& z_{i} \in\{0,1\}, \quad i=1, \ldots, N \\
& x_{i} \geq 0, \quad i=1, \ldots, N
\end{array}
$$

where $N$ is the number of assets in the portfolio, $x_{i}$ is the proportion of the total budget invested in asset $i$, and the constraint $\sum_{i=1}^{N} x_{i}=1$ ensures that the total budget is invested. Also, $\mu_{i}$ is the expected return of the $i$ th asset $(i=1, \ldots, N), \sigma_{i}$ denotes the standard deviation of assets, for $i \neq j$, and $\sigma_{i, j}$ is the covariance between assets $i$ and $j,(i=1, \ldots, N ; j=1, \ldots, N)$. The constraint $\sum_{i=1}^{N} z_{i}=K$, is the cardinality constraint which limits the number of assets held in the portfolio to $K$, and $\varepsilon_{i}$ and $\delta_{i}$ represent the lower and upper bounds of the $i$ th asset, respectively. Also, $\lambda \in[0,1]$ is the risk aversion parameter. When $\lambda=0$, the maximum expected return is obtained for the portfolio (regardless of the variance). In contrast, when $\lambda=1$ the minimum risk is found for the portfolio (regardless of the mean return). Therefore, the sensitivity of the investor to the risk decreases when $\lambda$ approaches zero, while it increases as $\lambda$ approaches unity. 
Short selling of assets is usually done by borrowing the assets under consideration and selling them. It leads to a portfolio with possibly better performance compared with the standard portfolio. The proceeds of a short selling are used as collateral for the lender of the asset and investor has to lend assets of the same value to the lender. In portfolio optimization models, when short selling is allowed, negative $x_{i}$ 's are interpreted as short positions. Thus the CCMV model (1) with short selling is as follows:

$$
\begin{aligned}
\min _{x, z} & \lambda\left(\sum_{i=1}^{N} \sum_{j=1}^{N} x_{i} x_{j} \sigma_{i, j}\right)-(1-\lambda)\left(\sum_{i=1}^{N} x_{i} \mu_{i}\right) \\
\text { s.t. } & \sum_{i=1}^{N} x_{i}=1, \\
& \sum_{i=1}^{N} z_{i}=K, \\
& \varepsilon_{i} z_{i} \leq x_{i} \leq \delta_{i} z_{i}, \quad i=1, \ldots, N \\
& z_{i} \in\{0,1\}, \quad i=1, \ldots, N,
\end{aligned}
$$

where $\varepsilon_{i}$ is negative.

Another factor that can be used with short selling strategy is the risk-neutral interest rate. Using it in the model may increase the return on the portfolio. Jacobs et al. in [14] used short selling and risk-neutral interest rate in their model and assumed that the portfolio includes $2 N$ stocks and $\sum_{i=1}^{2 N} x_{i}=1$, where $x_{i}$ represents a long position for $i \in[1, N]$, and a short position for $i \in[N+1,2 N]$. Dynamic of their model is

$$
\begin{array}{ll}
\min & \sum_{i=1}^{2 N} \sum_{j=1}^{2 N} x_{i} x_{j} \sigma_{i j} \\
\text { s.t. } & \sum_{i=1}^{N} r_{i} x_{i}+\sum_{i=N+1}^{2 N}\left(-r_{i-N}\right) x_{i}+\sum_{i=N+1}^{2 N} h_{i-N} r_{c} x_{i} \geq r_{e}, \\
& \sum_{i=1}^{2 N} x_{i}=1, \quad i=1,2, \cdots 2 N \\
& x_{i} \geq 0, \quad i=1, \ldots, 2 N
\end{array}
$$

where $r_{e}$ is the desired return and $r_{c}$ denotes risk-neutral interest rate. The term $r_{c} \sum_{i=N+1}^{2 N} h_{i-N} x_{i}$ represents the short rebate, where $0 \leq h_{i} \leq 1, \quad \forall i$.

Later, the authors in [25], applied a different form of model (3) as follows that does not specify short and long stocks in advance:

$$
\begin{array}{ll}
\min & \sum_{i=1}^{N} \sum_{j=1}^{N} x_{i} x_{j} \sigma_{i j} \\
\text { s.t. } & \sum_{i=1}^{N}\left(\mu_{i}-h_{i} r_{c}\right) x_{i} \geq r_{e}, \\
& \sum_{i=1}^{N} x_{i}=1, \quad-1<x_{i}<1, \quad i=1,2, \cdots N, \\
& \text { if } x_{i} \geq 0, \text { then } h_{i}=0, \\
& \text { if } \quad x_{i}<0, \text { then } 0<h_{i}<1 .
\end{array}
$$


Following model (4), by adding the risk-neutral interest rate to the CCMV model, we get the following:

$$
\begin{aligned}
\min _{x, z} & \lambda\left(\sum_{i=1}^{N} \sum_{j=1}^{N} x_{i} x_{j} \sigma_{i, j}\right)-(1-\lambda)\left(\sum_{i=1}^{N} x_{i} \mu_{i}\right)+(1-\lambda)\left(r_{c} \sum_{i=1}^{N} h_{i} x_{i}\right) \\
\text { s.t. } & \sum_{i=1}^{N} x_{i}=1, \\
& \sum_{i=1}^{N} z_{i}=K, \\
& \varepsilon_{i} z_{i} \leq x_{i} \leq \delta_{i} z_{i}, \quad i=1, \ldots, N, \\
& z_{i} \in\{0,1\}, \quad i=1, \ldots, N, \\
& \text { if } x_{i} \geq 0, \text { then } \quad h_{i}=0, \\
& \text { if } x_{i}<0, \text { then } \quad h_{i}=c, 0<c<1 .
\end{aligned}
$$

Using 0-1 variables, this model is equivalent to

$$
\begin{aligned}
\min _{x, z, p, w} & \lambda\left(\sum_{i=1}^{N} \sum_{j=1}^{N} x_{i} x_{j} \sigma_{i, j}\right)-(1-\lambda)\left(\sum_{i=1}^{N} x_{i} \mu_{i}\right)+(1-\lambda)\left(r_{c} \sum_{i=1}^{N} p_{i}\right) \\
\text { s.t. } & \sum_{i=1}^{N} x_{i}=1, \\
& \sum_{i=1}^{N} z_{i}=K, \\
& \varepsilon_{i} z_{i} \leq x_{i} \leq \delta_{i} z_{i}, \quad i=1, \ldots, N \\
& p_{i}-M w_{i} \leq 0, \quad i=1, \ldots, N \\
& -c x_{i}+p_{i} \leq 0, \quad i=1, \ldots, N \\
& c x_{i}-p_{i}+M w_{i} \leq M, \quad i=1, \ldots, N, \\
& p_{i}+M w_{i} \geq 0, \quad i=1, \ldots, N \\
& x_{i} \leq M\left(1-w_{i}\right), \quad i=1, \ldots, N \\
& x_{i} \geq-M w_{i}, \quad i=1, \ldots, N \\
& z_{i} \in\{0,1\}, \quad i=1, \ldots, N \\
& w_{i} \in\{0,1\}, \quad i=1, \ldots, N
\end{aligned}
$$

where $M$ is a large positive number. As one can see, model (5) has $2 N$ zero-one variables and $8 N+2$ constraints that might lead to a computationally inefficient model. In the sequel, we utilize an example to illustrate a drawback of model (5), when $\varepsilon_{i}=-\frac{1}{K}$ and $\delta_{i}=\frac{1}{K}$.

\section{Example 1}

Consider the data set of S\&P 500 index, Communication Service ${ }^{\dagger}$, for 2016 as given in Table 1. The results of solving model (5) for different number of assets are reported in Table 2. As we see, this model does not select assets in the short position (column 3) and also, if $K$ (the number of assets) is greater than the number of assets in the long position, it gives assets in short position with the positive proportion of investment (column 4). This means, in both cases, short selling is ineffective and model leads to unrealistic optimal objective values, returns

\footnotetext{
†https://finance.yahoo.com
} 
Table 1. Returns of Communication Service 2016 data

\begin{tabular}{cc}
\hline Company & 2016 \\
\hline Activision Blizzard & -0.0401 \\
CBS Corp & 0.3638 \\
Charter Communications & 0.6218 \\
Comcast Corp & 0.241 \\
Century Link Inc & -0.053 \\
AT\&T Inc & 0.2381 \\
Trip Advisor & -0.4412 \\
Take-Two Interactive & 0.4425 \\
Dish Network & -0.0401 \\
Omnicom Group & 0.1568 \\
\hline
\end{tabular}

Table 2. Results of model (5).

\begin{tabular}{cccc}
\hline Company & Return & $K=4$ & $K=8$ \\
\hline Activision Blizzard & -0.0401 & 0 & 0.125 \\
CBS Corp & 0.3638 & 0.25 & 0.125 \\
Charter Communications & 0.6218 & 0.25 & 0.125 \\
Comcast Corp & 0.241 & 0.25 & 0.125 \\
Century Link Inc & -0.053 & 0 & 0.125 \\
AT\&T Inc & 0.2381 & 0 & 0.125 \\
Trip Advisor & -0.4412 & 0 & 0 \\
Take-Two Interactive & 0.4425 & 0.25 & 0.125 \\
Dish Network & -0.0401 & 0 & 0 \\
Omnicom Group & 0.1568 & 0 & 0.125 \\
\hline
\end{tabular}

and risks. Motivated by this example, in the next section we present an improved variant of model (5) that has less $0-1$ variables and constraints compared to it, thus computationally efficient.

\section{Improved model}

Consider the following model instead of the CCMV model, where returns are replaced by absolute returns:

$$
\begin{array}{ll}
\min _{x, z} & \lambda\left(\sum_{i=1}^{N} \sum_{j=1}^{N} x_{i} x_{j} \sigma_{i, j}\right)-(1-\lambda)\left(\sum_{i=1}^{N} x_{i}\left|\mu_{i}\right|\right) \\
\text { s.t. } & \sum_{i=1}^{N} x_{i}=1, \\
& \sum_{i=1}^{N} z_{i}=K, \\
& 0 \leq x_{i} \leq \delta_{i} z_{i}, \quad i=1, \ldots, N, \\
& z_{i} \in\{0,1\}, \quad i=1, \ldots, N .
\end{array}
$$

In this model the constraint $\varepsilon_{i} z_{i} \leq x_{i}$ in model (2) is replaced by $0 \leq x_{i}$. The results of using model (6) for Example 1 are reported in Table 3. In this case, after solving the model, we replaced negative proportion of investment for assets in short selling position (short selling position before using absolute return).

\section{Lemma 1}

Let $Z^{*}$ and $W^{*}$ be the optimal objective values of models (1) and (6), respectively. Then $Z^{*} \geq W^{*}$. 
Table 3. Results of model (6).

\begin{tabular}{cccc}
\hline Company & Return & $K=4$ & $K=8$ \\
\hline Activision Blizzard & -0.0401 & 0 & 0 \\
CBS Corp & 0.3638 & 0.25 & 0.125 \\
Charter Communications & 0.6218 & 0.25 & 0.125 \\
Comcast Corp & 0.241 & 0 & 0.125 \\
Century Link Inc & -0.053 & 0 & 0 \\
AT\&T Inc & 0.2381 & 0 & 0.125 \\
Trip Advisor & -0.4412 & -0.25 & -0.125 \\
Take-Two Interactive & 0.4425 & 0.25 & 0.125 \\
Dish Network & -0.0401 & 0 & -0.125 \\
Omnicom Group & 0.1568 & 0 & 0.125 \\
\hline
\end{tabular}

\section{Proof}

Let $x^{*}$ be an optimal solution of model (1). Then

$$
\lambda\left(x^{*}\right)^{t} \Sigma x^{*}-(1-\lambda)\left(x^{*}\right)^{t} \mu \geq \lambda\left(x^{*}\right)^{t} \Sigma x^{*}-(1-\lambda)\left(x^{*}\right)^{t}|\mu| .
$$

Now let $\bar{x}$ be an optimal solution of model (6), since $x^{*}$ is also feasible for (6) we have

$$
\lambda\left(x^{*}\right)^{t} \Sigma x^{*}-(1-\lambda)\left(x^{*}\right)^{t}|\mu| \geq \lambda \bar{x}^{t} \Sigma \bar{x}-(1-\lambda)(\bar{x})^{t}|\mu| .
$$

Therefore $Z^{*} \geq W^{*}$.

To include more realistic features in model (6), we add risk-neutral interest rate as follows:

$$
\begin{array}{ll}
\min _{x, z} & \lambda\left(\sum_{i=1}^{N} \sum_{j=1}^{N} x_{i} x_{j} \sigma_{i, j}\right)-(1-\lambda)\left(\sum_{i=1}^{N} x_{i}\left|\mu_{i}\right|\right)-(1-\lambda)\left(r_{c} \sum_{i=1}^{N} h_{i} x_{i}\right) \\
\text { s.t. } & \sum_{i=1}^{N} x_{i}=1, \\
& \sum_{i=1}^{N} z_{i}=K, \\
& 0 \leq x_{i} \leq \delta_{i} z_{i}, \quad i=1, \ldots, N, \\
& z_{i} \in\{0,1\}, \quad i=1, \ldots, N,
\end{array}
$$

where $h$ is determined according to the $\mu$ values. If $\mu_{i} \geq 0$, then $h_{i}=0$ and if $\mu_{i}<0$, then the asset $i$ is in the short selling position thus $0 \leq h_{i} \leq 1$. As we see, no further $0-1$ variables are needed to include risk-neutral interest rate in model unlike model (5). It is also obvious that model (7) has better objective values compared to models (1) and (6).

\section{Numerical results}

In this section, we compare the returns and Sharpe ratios of models (1)-(7). We use the data set of the MIBTEL index (the Italian stock market index) from March 2003 until March 2008, and the data set of the S\&P 500 index, Information Technology ${ }^{\ddagger}$, for 2018. The results are reported for different $K$ (number of asset) values in Tables 4-7 for $\lambda=0.5, c=0.1, r_{c}=0.01$ and $\delta_{i}=-\varepsilon_{i}=\frac{1}{K}$ (the lower and upper bounds of the proportion of investment in any asset). All computations are performed in MATLAB R2017a on a $2.50 \mathrm{GHz}$ laptop with $4 \mathrm{~GB}$ of RAM. To solve the models, we used CVX 2.2 software using MATLAB [13]. 
Table 4. Returns of models (1)-(7) for Information Technology with 48 assets for $\lambda=0.5$

\begin{tabular}{ccccccc}
\hline Number of asset & model (1) & model (2) & model (5) & model (6) & model (7) & Naive strategy \\
\hline $\mathrm{k}=5$ & 0.4605 & 0.4605 & 0.4605 & 0.5246 & 0.5250 & - \\
$\mathrm{k}=10$ & 0.3481 & 0.3481 & 0.3481 & 0.4282 & 0.4288 & - \\
$\mathrm{k}=20$ & 0.2271 & 0.2271 & 0.2271 & 0.3327 & 0.3331 & - \\
$\mathrm{k}=30$ & 0.1444 & 0.1444 & 0.1444 & 0.2613 & 0.2617 & - \\
$\mathrm{k}=48$ & 0.0102 & 0.0102 & 0.0102 & 0.1816 & 0.1821 & 0.0102 \\
\hline
\end{tabular}

Table 5. Sharpe ratios of models (1)-(7) for Information Technology with 48 assets for $\lambda=0.5$

\begin{tabular}{ccccccc}
\hline Number of asset & model (1) & model (2) & model (5) & model (6) & model (7) & Naive strategy \\
\hline $\mathrm{k}=5$ & 0.1230 & 0.1230 & 0.1230 & 0.1528 & 0.1529 & - \\
$\mathrm{k}=10$ & 0.1017 & 0.1017 & 0.1017 & 0.1613 & 0.2618 & - \\
$\mathrm{k}=20$ & 0.0771 & 0.0771 & 0.0771 & 0.2718 & 0.2723 & - \\
$\mathrm{k}=30$ & 0.0531 & 0.0531 & 0.0531 & 0.1993 & 0.2998 & - \\
$\mathrm{k}=48$ & 0.0037 & 0.0037 & 0.0037 & 0.1671 & 0.1679 & 0.0037 \\
\hline
\end{tabular}

Table 6. Returns of models (1)-(7) and naive strategy for MIBTEL data with 226 assets for $\lambda=0.5$.

\begin{tabular}{ccccccc}
\hline Number of asset & model (1) & model (2) & model (5) & model (6) & model (7) & Naive strategy \\
\hline $\mathrm{K}=10$ & 0.0113 & 0.0113 & 0.0113 & 0.0114 & 0.0118 & - \\
$\mathrm{K}=20$ & 0.0096 & 0.0096 & 0.0096 & 0.0099 & 0.0100 & - \\
$\mathrm{K}=30$ & 0.0089 & 0.0089 & 0.0089 & 0.0091 & 0.0330 & - \\
$\mathrm{K}=40$ & 0.0084 & 0.0084 & 0.0084 & 0.0086 & 0.0087 & - \\
$\mathrm{K}=50$ & 0.0080 & 0.0080 & 0.0080 & 0.0082 & 0.0083 & - \\
$\mathrm{K}=100$ & 0.0066 & 0.0066 & 0.0066 & 0.0068 & 0.0069 & - \\
$\mathrm{K}=150$ & 0.0056 & 0.0056 & 0.0056 & 0.0058 & 0.0059 & - \\
$\mathrm{K}=200$ & 0.0046 & 0.0046 & 0.0046 & 0.0048 & 0.0050 & - \\
$\mathrm{K}=226$ & 0.0037 & 0.0037 & 0.0037 & 0.0045 & 0.0046 & 0.0037 \\
\hline
\end{tabular}

Table 7. Sharp ratios of models (1)-(7) and naive strategy for MIBTEL data with 226 assets for $\lambda=0.5$.

\begin{tabular}{ccccccc}
\hline Number of asset & model (1) & model (2) & model (5) & model (6) & model (7) & Naive strategy \\
\hline $\mathrm{K}=10$ & 0.0019 & 0.0019 & 0.0019 & 0.0020 & 0.0021 & - \\
$\mathrm{K}=20$ & 0.0021 & 0.0021 & 0.0021 & 0.0022 & 0.0025 & - \\
$\mathrm{K}=30$ & 0.0020 & 0.0020 & 0.0020 & 0.0021 & 0.0025 & - \\
$\mathrm{K}=40$ & 0.0020 & 0.0020 & 0.0020 & 0.0021 & 0.0024 & - \\
$\mathrm{K}=50$ & 0.0020 & 0.0020 & 0.0020 & 0.0021 & 0.0022 & - \\
$\mathrm{K}=100$ & 0.0019 & 0.0019 & 0.0019 & 0.0020 & 0.0022 & - \\
$\mathrm{K}=150$ & 0.0017 & 0.0017 & 0.0017 & 0.0019 & 0.0021 & - \\
$\mathrm{K}=200$ & 0.0014 & 0.0014 & 0.0014 & 0.0018 & 0.0019 & - \\
$\mathrm{K}=226$ & 0.0011 & 0.0011 & 0.0011 & 0.0018 & 0.0019 & 0.0011 \\
\hline
\end{tabular}

As we see in columns 2-4 of Tables 4-7, including short selling and risk-neutral interest rate in model (1) does not make any change in the returns and Sharpe ratios compared to model (1), while the new approach (columns 5 and 6) improves them. Also, by comparing models (5) and (7) when $K=N$, with the naive strategy in terms of returns and Sharpe ratios in Tables 4-7, we can see that model (5) and the naive strategy have the same results, while the proposed model (7) outperforms both of them.

We also compare the performance of all models (1), (5) and (7) using out of sample portfolio Sharpe ratio by employing Information Technology of S\&P index data on 2015-2017 which is taken from https://finance.yahoo.com with rolling-horizon procedure [6]. The results are reported in Table 8 when $\lambda=0.5$, $N=48, K=10,20, c=0.1, r_{c}=0.01$ and $\delta_{i}=-\varepsilon_{i}=\frac{1}{K}$. We compute Sharpe ratio $(\widehat{S R})$ as follows: $\widehat{S R}=\frac{\widehat{\mu}}{\widehat{\sigma}}$,

where

\footnotetext{
${ }_{\ddagger}^{\ddagger}$ https://finance.yahoo.com
} 


$$
\widehat{\sigma}^{2}=\frac{1}{T-\tau-1} \sum_{t=\tau}^{T-1}\left(x_{t}^{\prime} r_{t+1}-\widehat{\mu}\right)^{2}, \widehat{\mu}=\frac{1}{T-\tau} \sum_{t=\tau}^{T-1}\left(x_{t}^{\prime} r_{t+1}\right),
$$

and $r_{t+1}$ denotes the asset return, $x_{t}$ is the portfolio weight at time $t, t=\tau, \tau+1, \ldots, T-1, T$ is the total number of returns in the data set and $\tau$ is the length of the estimation time window. In our experiments, we use monthly asset return data from $2 / 1 / 2015$ to $29 / 12 / 2017$ and an estimation window of $\tau=24$ data, corresponds to 2 years.

Table 8. Out of sample portfolio Sharpe ratios of models (1), (5) and (7) for Information Technology of S\&P index data with $\lambda=0.5$.

\begin{tabular}{ccc}
\hline model & $K=10$ & $K=20$ \\
\hline model (1) & 0.4198 & 0.5906 \\
model (5) & 0.4198 & 0.5906 \\
model (7) & 0.6188 & 0.6930 \\
\hline
\end{tabular}

As we see, models (1) and (5) have similar portfolio Sharpe ratios and both of them are lower than the Sharp ratios of model (7). As we saw, model (5) has much more zero-one variables and constraints compared to model (7). Thus here, we compare their CPU times for the data set of the MIBTEL and S\&P indexes from March 2003 until March 2008. The corresponding results are reported in Tables 9 and 10 when $\lambda=0.5, c=0.1, r_{c}=0.01$ and $\delta_{i}=-\varepsilon_{i}=\frac{1}{K}$, that show model (7) is solved significantly faster than model (5). Therefore, the improved model outperforms the classical models in terms of returns, Sharpe ratios and CPU times.

Table 9. CPU times of models (5) and (7) for MIBTEL data with 226 assets.

\begin{tabular}{ccc}
\hline Desired number of assets & model $(5)$ & model $(7)$ \\
\hline $\mathrm{K}=10$ & 24.72 & 2.96 \\
$\mathrm{~K}=20$ & 426.44 & 3.85 \\
$\mathrm{~K}=50$ & 450.12 & 5.06 \\
$\mathrm{~K}=100$ & $>1 e+03$ & 5.94 \\
$\mathrm{~K}=150$ & $>1 e+03$ & 6.01 \\
\hline
\end{tabular}

Table 10. CPU times of models (5) and (7) for S\&P index data with 476 assets.

\begin{tabular}{ccc}
\hline Desired number of assets & model (5) & model (7) \\
\hline $\mathrm{K}=10$ & 34.57 & 4.37 \\
$\mathrm{~K}=20$ & 495.25 & 5.85 \\
$\mathrm{~K}=50$ & 513.64 & 6.42 \\
$\mathrm{~K}=100$ & $>1 e+03$ & 6.46 \\
$\mathrm{~K}=200$ & $>1 e+03$ & 7.77 \\
$\mathrm{~K}=300$ & $>1 e+03$ & 7.84 \\
\hline
\end{tabular}

\section{Conclusions}

This paper investigated CCMV model with and without short selling and risk-neutral interest rate combined with naive-like strategy. A drawback of the model is discussed, then an improved variant of it is proposed which uses absolute returns instead of the returns. Numerical results on the real data of MIBTEL and S\&P indexes showed that in terms of CPU times, the improved model is better than the classical one. Also, the returns and Sharpe ratios of the improved model are better than the classical models and naive strategy. 


\section{Acknowledgments}

The authors would like to thank the editor and reviewer for their comments and suggestions and University of Guilan for partially supporting this research.

\section{REFERENCES}

1. D. Bertsimas and R. Shioda, Algorithm for cardinality-constrained quadratic optimization, Computational Optimization and Applications 43 (2009), pp. 1-22.

2. T.R. Bielecki and I. Jang, Portfolio optimization with a defaultable security, Asia-Pacific Financial Markets 13 (2006), pp. $113-127$.

3. D. Bienstock, Computational study of a family of mixed-integer quadratic programming problems, Mathematical Programming 74 (1996), pp. 121-140.

4. J.R. Birge and R.Q. Zhang, Risk-neutral option pricing methods for adjusting constrained cash flows, The Engineering Economist 44 (1999), pp. 36-49.

5. T.J. Chang, N. Meade, J.E. Beasley, and Y.M. Sharaiha, Heuristics for cardinality constrained portfolio optimisation, Computers \& Operations Research 27 (2000), pp. 1271-1302.

6. Z. Dai and F. Wen, A generalized approach to sparse and stable portfolio optimization problem, The Journal of Industrial \& Management Optimization 14 (2018), pp. 1651-1666.

7. V. DeMiguel, L. Garlappi, and R. Uppal, Optimal versus naive diversification: How inefficient is the 1/n portfolio strategy?, The Review of Financial Studies 22 (2007), pp. 1915-1953.

8. U. Derigs and N.H. Nickel, Meta-heuristic based decision support for portfolio optimization with a case study on tracking error minimization in passive portfolio management, OR Spectrum 25 (2003), pp. 345-378.

9. G. Dueck and P. Winker, New concepts and algorithms for portfolio choice, Applied Stochastic Models and Data Analysis 8 (1992), pp. $159-178$

10. P.A. Frost and J.E. Savarino, For better performance: Constrain portfolio weights, The Journal of Portfolio Management 15 (1988), p. 29.

11. J. Gao and D. Li, Cardinality constrained linear-quadratic optimal control, IEEE Transactions on Automatic Control 56 (2011), pp. $1936-1941$

12. J. Gao and D. Li, Optimal cardinality constrained portfolio selection, Operations Research 61 (2013), pp. 745-761.

13. M. Grant, S. Boyd, and Y. Ye, Cvx: Matlab software for disciplined convex programming, version 2.0 beta (2013).

14. B.I. Jacobs, K.N. Levy, and H.M. Markowitz, Portfolio optimization with factors, scenarios, and realistic short positions, Operations Research 53 (2005), pp. 586-599.

15. B.I. Jacobs, K.N. Levy, and H.M. Markowitz, Trimability and fast optimization of long-short portfolios, Financial Analysts Journal 62 (2006), pp. 36-46.

16. R. Jagannathan and T. Ma, Risk reduction in large portfolios: Why imposing the wrong constraints helps, The Journal of Finance 58 (2003), pp. 1651-1683.

17. J.D. Jobson and B. Korkie, Estimation for markowitz efficient portfolios, Journal of the American Statistical Association 75 (1980), pp. 544-554.

18. C.C. Kwan, Portfolio selection under institutional procedures for short selling: Normative and market-equilibrium considerations, The Journal of Banking \& Finance 21 (1997), pp. 369-391.

19. D. Li and W.L. Ng, Optimal dynamic portfolio selection: Multiperiod mean-variance formulation, Mathematical Finance 10 (2000), pp. 387-406.

20. D. Li, X. Sun, and J. Wang, Optimal lot solution to cardinality constrained meanvariance formulation for portfolio selection, Mathematical Finance 16 (2006), pp. 83-101.

21. J. Lintner, Security prices, risk, and maximal gains from diversification, The Journal of Finance 20 (1965), pp. 587-615.

22. D. Maringer, Portfolio Management With Heuristic Optimization, Springer Science \& Business Media, 2006.

23. D. Maringer and H. Kellerer, Optimization of cardinality constrained portfolios with a hybrid local search algorithm, OR Spectrum 25 (2003), pp. 481-495.

24. H. Markowitz, Portfolio selection, The Journal of Finance 7 (1952), pp. 77-91.

25. A.A. Najafi and H. reza Ghasemi, Portfolio optimization in terms of justifiability short selling and some market practical constraints, Financial Research Journal 14 (2013), pp. 117-132.

26. N.R. Patel and M.G. Subrahmanyam, A simple algorithm for optimal portfolio selection with fixed transaction costs, Management Science 28 (1982), pp. 303-314.

27. G.C. Pflug, A. Pichler, and D. Wozabal, The 1/n investment strategy is optimal under high model ambiguity, Journal of Banking \& Finance 36 (2012), pp. 410-417.

28. D.X. Shaw, S. Liu, and L. Kopman, Lagrangian relaxation procedure for cardinality-constrained portfolio optimization, Optimization Methods \& Software 23 (2008), pp. 411-420.

29. J. Xie, S. He, and S. Zhang, Randomized portfolio selection with constraints, Pacific Journal of Optimization 4 (2008), pp. 89-112.

30. S. Zhang, S. Wang, and X. Deng, Portfolio selection theory with different interest rates for borrowing and leading, Journal of Global Optimization 28 (2004), pp. 67-95. 